\title{
Randomized, Double-Blind, Placebo-Controlled Phase II Clinical Trial on the Use of Uncaria tomentosa (Cat's Claw) for Aromatase Inhibitor-Induced Arthralgia: A Pilot Study
}

\author{
Renan Sordi ${ }^{1}$, Suellen Nastri Castro ${ }^{1}$, Andrea Thaumaturgo Lera ${ }^{1}$, Macilon Nonato Irene ${ }^{1}$, \\ Marilia de Melo Farinazzo ${ }^{1}$, Claudia Sette ${ }^{2}$, Daniel de Iracema Gomes Cubero', \\ Ana Luisa de Castro Baccarin ${ }^{2}$ and Auro del Giglio ${ }^{2 *}$ \\ ${ }^{1}$ Medical Residents of Clinical Oncology of the Brazilian Institute of Cancer Control \\ 2Department of Clinical Oncology, Brazilian Institute of Cancer Control; and Hematology and \\ Oncology Sector, School of Medicine of ABC, Principe de Gales Avenue, 821, Santo \\ Andre - SP - 09060-650, Brazil; aurodelgiglio@gmail.com
}

\begin{abstract}
Introduction: Aromatase inhibitors (AI) are widely used in adjuvant breast cancer treatment, and diffuse articular pain secondary to AI use is the most common cause of treatment discontinuation. Uncaria tomentosa (cat's claw) has antiinflammatory activity and is used to treat arthrosis and arthritis. Patients and Methods: This prospective, single-center, double-blind, randomized, placebo-controlled phase II study analyzed 70 patients with breast cancer undergoing AI therapy with complaints of arthralgia. The patients received $100 \mathrm{mg}$ of the dry extract of Uncaria tomentosa three times daily for 4 weeks. At the beginning and end of the study, patients answered the Brief Pain Inventory (BPI), Disability Arm, Shoulder, and Hand (DASH), Lequesne, SF-36 Quality of Life questionnaires, completed a visual analog scale (VAS) for pain, and underwent laboratory testing. Results: $U$. tomentosa was not more effective than the placebo. No evidence of grade 3 or 4 toxicity was found. In addition, no significant differences were seen in laboratory results or inflammatory markers between the two study groups. Conclusion: Dry extract of $U$. tomentosa was safe but ineffective in reducing AI-induced arthralgia compared with the placebo. Furthermore, the plant extract had no detectable anti-inflammatory activity.
\end{abstract}

Keywords: Aromatase Inhibitor, Arthralgia, Cat's Claw, Uncaria tomentosa

\section{Introduction}

Aromatase inhibitors (AIs) are widely used in adjuvant breast cancer treatment in postmenopausal women with positive hormone receptors on immunohistochemistry. AIs inhibit the enzyme aromatase, reducing estrogen production by blocking the conversion of androgen to estrogen, and are considered the most effective adjuvant hormone therapy available for these patient groups ${ }^{1}$.

Studies have demonstrated that AIs improved diseasefree survival and decreased the rate of contralateral breast cancer compared with adjuvant tamoxifen ${ }^{1}$. However, nearly $50 \%$ of patients with indications for AIs use do not adequately adhere to treatment, and the discontinuation rate of AIs use in the first year of treatment is approximately $20 \%$. Arthralgia is the most common reason for poor adherence and discontinued treatment ${ }^{2}$.

The onset of AI-induced arthralgia occurs approximately 2 months after initiating treatment and peaks at approximately 6 months of treatment; however, this disease may recur up to 2 years after initiating 
therapy ${ }^{2}$. It is characterized by symmetrical joint pain, usually in the wrists, metacarpals, and knees. Specific diagnostic criteria for AI-induced arthralgia have been established ${ }^{2}$. The occurrence of AI-induced arthralgia affects patients quality of life and may be responsible for poor treatment adherence ${ }^{2}$.

Uncaria tomentosa is a plant species found in the Amazon region and is widely distributed in South and Central America. This species belongs to the family Rubiaceae and subfamily Cinchonoideae, and its popular name, "cat's claw", is derived from the resemblance of the plant's thorns to claws. This plant species was used by the Incas for centuries to treat arthritis, arthrosis, viral infections, cancer, and other inflammatory conditions because its active metabolites, including pentacyclic and indole oxindole alkaloids and quinovic acid glycosides, have antioxidant, immunomodulatory, antineoplastic, anti-inflammatory, and antiviral activities ${ }^{3-4}$. The antiinflammatory effects of $U$. tomentosa may be related to the decreased expression of transcription factor NFkbeta, which decreases tumor necrosis factor (TNF) levels, leading to increased anti-inflammatory activity in cells ${ }^{3}$.

The primary objective of this study is to evaluate arthralgia reduction by $U$. tomentosa dry extract in patients with breast cancer in adjuvant treatment or chemoprophylaxis of mammary carcinoma in situ who developed arthralgia during AI use or whose previous arthralgia symptoms intensified with AI use, using specific questionnaires to assess joint pain and quality of life.

The secondary objectives were to evaluate the drug's safety and to determine the relationship between arthralgia and inflammatory markers.

\section{Materials and Methods}

\subsection{Study Design}

This prospective, single-center, double-blind, Uncaria tomentosa dry extract versus placebo, phase II study included patients using AI who developed arthralgia during cancer treatment or whose previous symptoms of arthralgia intensified with AI use.

This study was approved by the Research Ethics Committee of the Instituto Brasileiro de Controle do
Cancer (IBCC). Patients were recruited and evaluated in the IBCC outpatient clinics.

\subsection{Inclusion and Exclusion Criteria}

Inclusion criteria were postmenopausal women with a history of breast cancer and/or carcinoma in situ, treated in IBCC outpatient clinics, with positive hormone receptors on immunohistochemistry, undergoing adjuvant AI and/or chemoprophylaxis for at least 2 months, who developed moderate or severe arthralgia after initiating AI treatment or whose arthralgia worsened by $40 \%$ or with a score $\geq 5$ on the Brief Pain Inventory (BPI) questionnaire.

Exclusion criteria were women diagnosed with or treated for diseases that cause arthralgia such as rheumatoid arthritis, arthrosis, or fibromyalgia, or arthralgia before using anastrozole, pregnant or breastfeeding women, transplanted patients, and patients with anemia at the time of recruitment.

Patients who failed to take the medication for a 48-hour period or developed severe adverse events from drug therapy were excluded from the study.

\subsection{Study Procedures}

After patients signed the consent form, their clinical histories, analgesic use, and physical activity were evaluated. During the same visit, serum biological samples were collected for laboratory tests, and pain levels were assessed using the BPI, Lequesne, Disability Arm, Shoulder, and Hand (DASH), and SF-36 Quality of Life questionnaires.

Treatment lasted 4 weeks. Between weeks 2 and 3, treatment adherence and side effects were evaluated by phone. At week 4, patients had a new consultation with the investigator to collect biological material for laboratory testing and to reassess pain and quality of life with the questionnaires.

A daily record of medication use, physical activity, and improvement of pain or other symptoms was provided to each patient at the first visit and collected by the investigator at the end of the study.

\subsection{Efficacy Parameters}

The efficacy parameters in this study were classified using five tools: 
- Brief Pain Inventory (BPI) $)^{5}$

- Visual analog scale (VAS) for pain ${ }^{6}$

- Lequesne (osteoarthritis) ${ }^{7}$

- Disabilities of the Arm, Shoulder, and Hand (DASH) ${ }^{8}$

- SF-36 questionnaire for assessing the quality of life ${ }^{9}$

\subsection{Drug Therapy}

The drug used was $U$. tomentosa dry extract in manipulated $100-\mathrm{mg}$ capsules standardized to $5.0+$ $0.5 \%$ total alkaloids expressed as mitraphylline (Lot TYC160110, manufactured on January 10, 2016 and valid until January 9, 2019).

The placebo was manipulated in 100-mg capsules identical to the $U$. tomentosa capsules manufactured using cornstarch and food coloring.

The dosage was 100-mg tablet of the dry extract three times daily for 30 days, as recommended in the package insert.

Treatment adherence was evaluated by counting the number of capsules in the returned packages. For this purpose, the patients were instructed to return the packages to the investigator at the end of the study and the packages were then returned to the pharmacy.

\subsection{Laboratory Tests}

Whole blood $(15 \mathrm{~mL})$ was collected from each patient at the first and last visits. The blood was transferred to two collection tubes containing EDTA and two collection tubes containing cobalt gel. Laboratory analyses included a complete blood count, erythrocyte sedimentation rate (ESR), C-reactive protein (CRP), albumin, estradiol, and vitamin D. All tests were performed in duplicate at the Laboratory of Clinical Analysis of FMABC following good clinical practices.

The two patient groups were biochemically compared using the Glasgow Prognostic Score (GPS) ${ }^{10}$ based on serum CRP and albumin levels and neutrophillymphocyte ratios. The first classification considered patients with a low risk of systemic inflammation as normal CRP $(<10 \mathrm{mg} / \mathrm{L})$ regardless of albumin level; patients with an intermediate risk as high CRP $(>10$ $\mathrm{mg} / \mathrm{L})$ and normal albumin $(5 \mathrm{~g} / \mathrm{L})$; and patients with a high risk as high CRP $(>10 \mathrm{mg} / \mathrm{L})$ and hypoalbuminemia $(<3.5 \mathrm{~g} / \mathrm{L})$. The second classification using the lymphocyte ratio was determined by dividing the total number of neutrophils by the total number of lymphocytes.

\subsection{Adverse Events}

Patients were asked to report adverse events to the investigator and were scored using the toxicity criteria developed by the National Cancer Institute, version 2.0 $0^{11}$

\subsection{Statistical Analysis}

The sample size was calculated by considering an intergroup difference in arthralgia reduction of $40 \%$, type 1 error of 0.05 , and power of $80 \%$. The analysis predicted the inclusion of 35 patients per group.

We evaluated associations between continuous and discrete variable though the ANOVA test. Associations between discrete variables were evaluated by the Chisquare test or the Fisher Exact test.

All statistical analyses, concerning questionnaire data, were conducted using the NCSS statistical package (https://www.ncss.com/software/ncss/).

\section{Results}

\subsection{Study Population}

Seventy patients were randomized from October 1, 2016, to March 1, 2017 (Figure 1). The mean age was 59.2 years, all patients were women, and $58 \%$ were Caucasian. Clinical stage I was observed in $29.8 \%$ of patients, and luminal subtype B was the most prevalent on immunohistochemistry (49.3\%). Most patients $(66.3 \%)$ had undergone chemotherapy, and of these, $51.9 \%$ had undergone taxane therapy. Anastrozole was used in $98.7 \%$ of patients and exemestane was used in one patient (1.3\%). The mean AI therapy length was 20.58 months (2-96 months). Of the 70 patients, $13(16.8 \%)$ performed aerobic physical activity, and 49 (70\%) used analgesics to manage arthralgia. The most common painful regions were the knees $(57 ; 81.42 \%)$, spine ( 45 ; $64.28 \%)$, metacarpus $(43 ; 61.42 \%)$, and shoulders (34; 48.57\%). The mean BMI was $29.73 \pm 5.69$. The patients' general characteristics are presented in Table 1. 

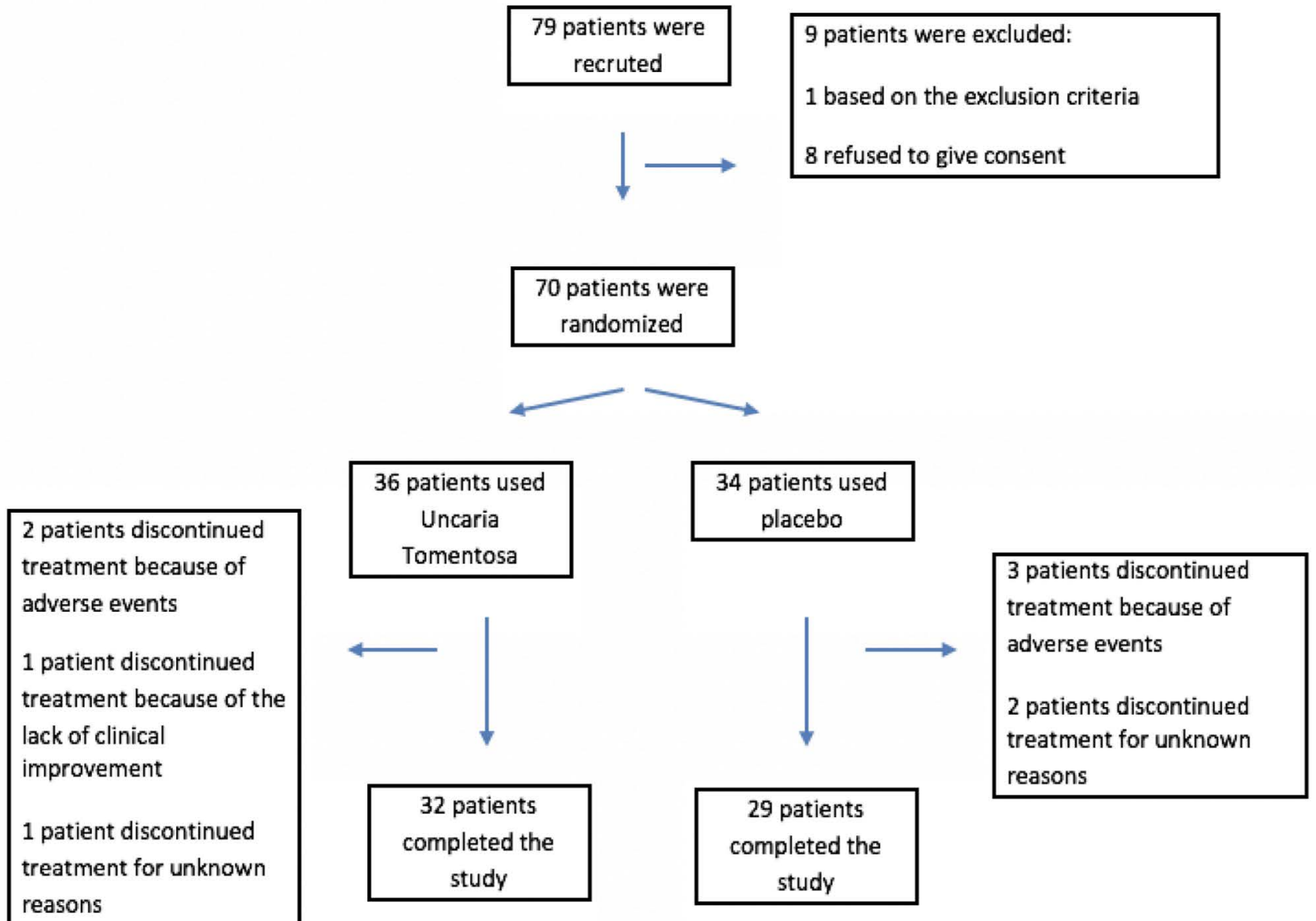

Figure 1. Study flowchart.

\subsection{Evaluation of the Questionnaires and Hematological and Inflammatory Parameters}

$U$. tomentosa dry extract was not more effective than the placebo when evaluated using the questionnaires. On the VAS for assessing pain intensity, the placebo was more effective than the dry extract $(\mathrm{p}=0.02)$ (Table 2 ). All patients who completed the study answered the questionnaires.

Similarly, no significant differences were seen in inflammatory or hematological markers between the dry extract and the placebo (Tables 3 and 4). Laboratory analysis was conducted for only 50 patients (25 in the placebo group and 25 in the control group) because some patients who completed the study had their second blood sample collected more than 3 months after starting the drug or placebo treatments.
Use of $U$. tomentosa in this patient group was safe, and no cases of grade 3 or 4 toxicity occurred (Table 5). Adverse events were analyzed in 32 patients who underwent AI therapy and completed the study, two patients who discontinued AI therapy because of adverse events, 29 patients who used a placebo and completed the study and three patients who discontinued placebo treatment because of adverse events.

\section{Discussion}

Several hypotheses may explain the etiology of AI-induced arthralgia, particularly the hypothesis that AI intensifies low estrogen levels during menopause.

Estrogen may exert antinociceptive activity. Higher estrogen levels suppress inflammatory cytokine production, whereas lower levels increase cytokine production ${ }^{12-13}$. In addition, estrogen 
deficiency increases cartilage turnover, thereby reducing its protective capacity ${ }^{2}$ the figure has been much higher in subsequent case series. There is concern that these symptoms are significant and may affect compliance and thus the overall efficacy of treatment. It is therefore extremely important that we evaluate this syndrome with a view to gaining more information regarding its clinical features and possible aetiological mechanism. The potential aetiological mechanisms and evidence for aromatase inhibitor-induced arthralgia (AIA).

These results and $U$. tomentosa's reported anti-inflammatory activity for chronic inflammatory diseases, including rheumatoid arthritis and arthrosis in phase II studies ${ }^{14}$, stimulated the investigation of this plant extract's efficacy in treating AI-induced arthralgia.

As previously reported in an uncontrolled study conducted by our research group ${ }^{15}$, no detectable effect of $U$. tomentosa was found on the inflammatory markers studied, including ultra-sensitive CRP, the ESR, or the GPS. Results from our previous study found no significant differences in serum levels of IL6, IL1, TNFa, or alpha-1-glycoprotein between, before or after $U$. tomentosatreatment ${ }^{15}$. Mur et al. ${ }^{14}$ found a modest reduction of arthralgia in patients

Table 1. Patient demographics

\begin{tabular}{|c|c|c|}
\hline & DRUG $(n=36)$ & PLACEBO $(n=34)$ \\
\hline Age, mean (range) & $59.2(33-79)$ & $60.79(43-80)$ \\
\hline BMI, mean (range) & $28.6(20-45)$ & $30.88(46-23)$ \\
\hline Use of Al (months), mean (range) & $23.97(72-2)$ & $17.5(96-2)$ \\
\hline$N(\%)$ & Race & Race \\
\hline Caucasian & $21(58 \%)$ & $24(70.5 \%)$ \\
\hline Mixed & $9(25 \%)$ & $4(11.7 \%)$ \\
\hline Black & $5(13.8 \%)$ & $6(17.6 \%)$ \\
\hline Asian & $1(2.7 \%)$ & $0(0 \%)$ \\
\hline $\mathbf{N}(\%)$ & Cancer staging & Cancer staging \\
\hline Carcinoma in situ & $0(0 \%)$ & $1(2.9 \%)$ \\
\hline I & $9(25 \%)$ & $14(41 \%)$ \\
\hline II & $16(44.4 \%)$ & $11(32.35 \%)$ \\
\hline III & $11(30.5 \%)$ & $8(23.5 \%)$ \\
\hline $\mathbf{N}(\%)$ & Immunohistochemistry & Immunohistochemistry \\
\hline Luminal A & $13(36.1 \%)$ & $10(29.4 \%)$ \\
\hline Luminal B & $21(58.3 \%)$ & $17(50 \%)$ \\
\hline Luminal hybrid & $2(5.5 \%)$ & $7(20.5 \%)$ \\
\hline \multirow[t]{2}{*}{ Previous chemotherapy } & $28(77.7 \%)$ & $23(67.6 \%)$ \\
\hline & Before treatment & After treatment \\
\hline Previous chemotherapy with taxanes & $21(58.3 \%)$ & $19(55.8 \%)$ \\
\hline Previous radiotherapy & $31(86.1 \%)$ & $29(85.2 \%)$ \\
\hline Physical exercise & $6(16.6 \%)$ & $7(20.5 \%)$ \\
\hline N (\%) & Current treatment with $\mathrm{AI}$ & Current treatment with $\mathrm{Al}$ \\
\hline Anastrozole & 35 (97.2\%) & 34 (100\%) \\
\hline Others & $1(2.7 \%)$ & $0(0 \%)$ \\
\hline
\end{tabular}


Table 2. Questionnaire evaluation

\begin{tabular}{|c|c|c|c|c|c|c|}
\hline & \multicolumn{3}{|c|}{ Before treatment } & \multicolumn{3}{|c|}{ After treatment } \\
\hline & Drug & Placebo & p-value & Drug & Placebo & p-value \\
\hline SF36 (functional capacity) & $25 \pm 26.9$ & $30 \pm 24.7$ & 0.93 & $40 \pm 23.8$ & $37.5 \pm 28.1$ & 0.72 \\
\hline SF36 (physical limitations) & $25 \pm 28.62$ & $0 \pm 35.8$ & 0.85 & $25 \pm 37.04$ & $50 \pm 43.75$ & 0.46 \\
\hline SF36 (pain) & $31 \pm 13.15$ & $36.5 \pm 18.5$ & 0.062 & $32 \pm 21.3$ & $41.5 \pm 26.1$ & 0.07 \\
\hline SF36 (overall health status) & $76.5 \pm 24.2$ & $86 \pm 25.1$ & 0.35 & $62 \pm 19$ & $63.5 \pm 26.2$ & 0.91 \\
\hline SF36 (vitality) & $40 \pm 19.86$ & $50 \pm 24.1$ & 0.11 & $45 \pm 22.3$ & $55 \pm 21.05$ & 0.096 \\
\hline SF36 (social aspects) & $56 \pm 31.8$ & $75 \pm 29.3$ & 0.04 & $62.5 \pm 30.4$ & $75 \pm 29.5$ & 0.21 \\
\hline SF36 (emotional aspects) & $33.79 \pm 39.70$ & $33 \pm 43.2$ & 0.61 & $66 \pm 38.9$ & $66.3 \pm 43.3$ & 0.52 \\
\hline SF36 (mental health) & $54 \pm 29.9$ & $64 \pm 21.6$ & 0.31 & $64 \pm 25.5$ & $68 \pm 25.3$ & 0.68 \\
\hline Lequesne & $15 \pm 6.2$ & $15.25 \pm 5.5$ & 0.87 & $12 \pm 6.2$ & $10 \pm 5.6$ & 0.4 \\
\hline DASH (disabilities) & $43.75 \pm 23.90$ & $33.12 \pm 24.70$ & 0.97 & $25 \pm 25.37$ & $23.75 \pm 27.10$ & 0.63 \\
\hline DASH (pain) & $62 \pm 25.5$ & $62 \pm 22.8$ & 0.87 & $48 \pm 28.7$ & $54 \pm 28.6$ & 0.69 \\
\hline BPI (pain severity) & $6.5 \pm 1.39$ & $6.375 \pm 8.3$ & 0.41 & $6.5 \pm 7.3$ & $5.25 \pm 2.7$ & 0.08 \\
\hline BPI (pain interference) & $5.75 \pm 7.5$ & $5 \pm 10.1$ & 0.83 & $3.4 \pm 7.8$ & $3.55 \pm 3.1$ & 0.3 \\
\hline VAS & $8 \pm 2.1$ & $8 \pm 2.1$ & 0.71 & $8 \pm 2.74$ & $5 \pm 3.1$ & 0.02 \\
\hline
\end{tabular}

Table 3. Laboratory analysis

\begin{tabular}{|l|c|c|c|c|c|c|}
\cline { 2 - 7 } \multicolumn{1}{c|}{} & \multicolumn{3}{c|}{ Before treatment } & \multicolumn{3}{c|}{ After treatment } \\
\cline { 2 - 7 } \multicolumn{1}{c|}{} & Drug & Placebo & p-value & Drug & Placebo & p-value \\
\hline Vitamin D & $31 \pm 9.03$ & $29 \pm 11.2$ & 0.85 & $31.1 \pm 9.8$ & $24 \pm 10.6$ & 0.5 \\
\hline Estradiol & $6 \pm 4.7$ & $6 \pm 0.45$ & 0.21 & $6 \pm 3.7$ & $6 \pm 1.5$ & 0.36 \\
\hline Erythrocyte sedimentation rate & $21 \pm 24.1$ & $23.5 \pm 13.7$ & 0.76 & $13 \pm 18.2$ & $19 \pm 17.5$ & 0.49 \\
\hline Albumin & $4.2 \pm 0.22$ & $4.2 \pm 0.29$ & 0.84 & $4.3 \pm 0.3$ & $4.3 \pm 0.3$ & 0.65 \\
\hline C-reactive protein & $6 \pm 7.86$ & $6 \pm 3.6$ & 0.43 & $6 \pm 1.8$ & $6 \pm 1.9$ & 0.59 \\
\hline Hemoglobin & $12.7 \pm 1.2$ & $13.3 \pm 0.9$ & 0.21 & $12.6 \pm 1.1$ & $13 \pm 10$ & 0.28 \\
\hline Neutrophil-lymphocyte ratio & $1.89 \pm 0.97$ & $1.93 \pm 0.85$ & 0.2 & $1.93 \pm 0.81$ & $1.62 \pm 0.69$ & 0.35 \\
\hline Platelets & $225 \pm 57.68$ & $220 \pm 46.5$ & 0.34 & $234.5 \pm 59.6$ & $213 \pm 66.3$ & 0.08 \\
\hline
\end{tabular}

Table 4. Glasgow Prognostic Score rating

\begin{tabular}{|l|c|c|c|c|}
\hline \multirow{2}{*}{} & \multicolumn{3}{|c|}{ Glasgow Prognostic Score } \\
\cline { 2 - 5 } & \multicolumn{2}{|c|}{ Before treatment } & \multicolumn{2}{c|}{ After treatment } \\
\hline & Drug & Placebo & Drug & Placebo \\
\hline Low risk & $24(88 \%)$ & $\begin{array}{c}24 \\
(92.3 \%)\end{array}$ & $\begin{array}{c}23 \\
(92 \%)\end{array}$ & $22(88 \%)$ \\
\hline $\begin{array}{l}\text { Intermediate } \\
\text { risk }\end{array}$ & $3(11.1 \%)$ & $2(7.29 \%)$ & $2(8 \%)$ & $3(12 \%)$ \\
\hline High risk & 0 & 0 & 0 & 0 \\
\hline Total & $27(100 \%)$ & $26(100 \%)$ & $\begin{array}{c}25 \\
(100 \%)\end{array}$ & $\begin{array}{c}25 \\
(100 \%)\end{array}$ \\
\hline
\end{tabular}

with rheumatoid arthritis treated with $U$. tomentosa but detected no significant changes in CRP or ESR in patients treated with $U$. tomentosa versus the placebo. Similarly, Araujo et al. ${ }^{16}$ who underwent a treatment regimen known as FAC (Fluorouracil, Doxorubicin, Cyclophosphamide)observed no significant changes in IL-6 or lymphocyte subpopulation levels in patients with breast carcinoma receiving adjuvant chemotherapy combined or not with $U$. tomentosa. The lack of a beneficial effect of $U$. tomentosa on AIinduced arthralgia in our study may be due to the 
Randomized, Double-Blind, Placebo-Controlled Phase II Clinical Trial on the Use of Uncaria tomentosa (Cat's Claw) for Aromatase Inhibitor-Induced Arthralgia: A Pilot Study

Table 5. Adverse Events

\begin{tabular}{|c|c|c|c|c|c|}
\hline \multicolumn{6}{|c|}{ PATIENTS USING DRUG $(n=34)$} \\
\hline & \multicolumn{5}{|c|}{ SEVERITY OF THE EVENT } \\
\hline ADVERSE EVENT & All grades & GRADE 1 & GRADE 2 & GRADE 3 & GRADE 4 \\
\hline Nausea & $1(2.94 \%)$ & 1 & 0 & 0 & 0 \\
\hline Vomiting & 0 & 0 & 0 & 0 & 0 \\
\hline Abdominal pain & $1(2.94 \%)$ & 1 & 0 & 0 & 0 \\
\hline Abdominal distension & 0 & 0 & 0 & 0 & 0 \\
\hline Constipation & $1(2.94 \%)$ & 1 & 0 & 0 & 0 \\
\hline Epigastralgia & 0 & 0 & 0 & 0 & 0 \\
\hline Diarrhea & $2(5.88 \%)$ & 2 & 0 & 0 & 0 \\
\hline Intestinal subocclusion & $1(2.94 \%)$ & 1 & 0 & 0 & 0 \\
\hline Lower limb edema & $1(2.94 \%)$ & 1 & 0 & 0 & 0 \\
\hline Neuropathy & $1(2.63 \%)$ & 1 & 0 & 0 & 0 \\
\hline Allergic reaction & 0 & 0 & 0 & 0 & 0 \\
\hline \multicolumn{6}{|c|}{ PATIENTS USING PLACEBO $(n=32)$} \\
\hline & \multicolumn{5}{|c|}{ SEVERITY OF THE EVENT } \\
\hline ADVERSE EVENT & All grades & GRADE 1 & GRADE 2 & GRADE 3 & GRADE 4 \\
\hline Nausea & $3(9.37 \%)$ & 0 & 3 & 0 & 0 \\
\hline Vomiting & $3(9.37 \%)$ & 2 & 1 & 0 & 0 \\
\hline Abdominal pain & $1(3.12 \%)$ & 1 & 0 & 0 & 0 \\
\hline Abdominal distension & $1(3.12 \%)$ & 1 & 0 & 0 & 0 \\
\hline Constipation & 0 & 0 & 0 & 0 & 0 \\
\hline Epigastralgia & $1(3.12 \%)$ & 0 & 1 & 0 & 0 \\
\hline Diarrhea & $3(9.37 \%)$ & 2 & 1 & 0 & 0 \\
\hline Intestinal subocclusion & 0 & 0 & 0 & 0 & 0 \\
\hline Lower limb edema & $1(3.12 \%)$ & 1 & 0 & 0 & 0 \\
\hline Neuropathy & $2(6.25 \%)$ & 2 & 0 & 0 & 0 \\
\hline Allergic reaction & 2 (6.25\%) & 2 & 0 & 0 & 0 \\
\hline
\end{tabular}

lack of a significant effect on inflammatory markers in the treated patients. In addition, as described above $^{2}$ the etiopathogenesis of AI-induced arthralgia appears to be multifactorial, and inflammation is one of several causes.

No significant changes were found in laboratory parameters in patients treated with $U$. tomentosa compared with the placebo group. Moreover, patients receiving $U$. tomentosa showed no grade 3 or 4 toxicity. Consistent with our previous study ${ }^{15}$, $U$. tomentosa was safe at the doses used in this study.

\section{Conclusion}

In conclusion, using $U$. tomentosa dry extract was safe but not more effective than the placebo in treating AI-induced arthralgia; therefore, use of this extract cannot be recommended for this purpose.

\section{References}

1. Conte P, Frassoldati A. Aromatase inhibitors in the adjuvant treatment of postmenopausal women with early breast cancer: Putting safety issues into perspective. The Breast Journal. 
2007 Feb; 13(1): 28-35. https://doi.org/10.1111/j.15244741.2006.00359.x PMid:17214790

2. Din OS, Dodwell D, Wakefield RJ, Coleman RE. Aromatase inhibitor-induced arthralgia in early breast cancer: what do we know and how can we find out more? Breast Cancer Research and Treatment. 2010 Apr; 120(3): 525-38. https:// doi.org/10.1007/s10549-010-0757-7 PMid:20157776t

3. Aguilar JL, Rojas P, Marcelo A, Plaza A, Bauer R, Reininger $\mathrm{E}$, et al. Anti-inflammatory activity of two different extracts of Uncaria tomentosa (Rubiaceae). Journal of Ethnopharmacology. 2002 Jul; 81(2): 271-6. https://doi. org/10.1016/S0378-8741(02)00093-4

4. Aquino R, De Simone F, Pizza C, Conti C, Stein ML. Plant metabolites. Structure and in vitro antiviral activity of quinovic acid glycosides from Uncaria tomentosa and Guettardaplatypoda. Journal of Natural Products. 1989 Aug; 52(4): 679-85. https://doi.org/10.1021/np50064a002 PMid:2553871

5. Ferreira-Valente MA, Pais Ribeiro JL, Jensen MP. Further Validation of a Portuguese Version of the Brief Pain Inventory Interference Scale. Clinica Salud [Internet]. 2012; 23(1). [Cited 2018 May 15]. Available from: http://www.redalyc.org/ resumen.oa?id=180623381005.

6. Measures of adult pain: Visual Analog Scale for Pain (VAS Pain), Numeric Rating Scale for Pain (NRS Pain), McGill Pain Questionnaire (MPQ), Short-Form McGill Pain Questionnaire (SF-MPQ), Chronic Pain Grade Scale (CPGS), Short Form-36 Bodily Pain Scale (SF-36 BPS), and Measure of Intermittent and Constant Osteoarthritis Pain (ICOAP) Hawker - 2011 - Arthritis Care and Research - Wiley Online Library [Internet]. [cited 2018 May 15]. Available from: https://onlinelibrary.wiley.com/doi/abs/10.1002/acr.20543.

7. Marx FC, Oliveira LM de, Bellini CG, Ribeiro MCC. Translation and cultural validation of the Lequesne's algofunctional questionnaire for osteoarthritis of knee and hip for portuguese language. Revista Brasileira De Reumatologia. 2006 Aug; 46(4): 253-60.

8. Moser AD de L, Knaut LAM, Zotz TG, Scharan KO. Validity and reliability of the portuguese version of the American Shoulder and Elbow Surgeons Standardized Shoulder Assessment Form. Revista Brasileira De Reumatologia.
2012 Jun; 52(3): 352-6. https://doi.org/10.1590/S048250042012000300005

9. Ciconelli RM, Ferraz MB, Santos W, Meinao I, Quaresma MR. Traducao para a lingua portuguesa e validacao do questionario generico de avaliacao de qualidade de vida SF-36 (Brasil SF-36). Revista Brasileira De Reumatologia. 1999 Jun; 39(3): 143-50.

10. McMillan, Donald C. The systemic inflammation-based Glasgow Prognostic Score: a decade of experience in patients with cancer. Cancer Treatment Reviews. 2013; 39(5): 534-40. https://doi.org/10.1016/j.ctrv.2012.08.003 PMid:22995477

11. Trotti A, Byhardt R, Stetz J, Gwede C, Corn B, Fu K, et al. Common toxicity criteria: version 2.0. an improved reference for grading the acute effects of cancer treatment: impact on radiotherapy. International Journal of Radiation Oncology, Biology, Physics. 2000 Apr 1; 47(1): 13-47. https://doi. org/10.1016/S0360-3016(99)00559-3

12. Cutolo M, Villaggio B, Seriolo B, Montagna P, Capellino $\mathrm{S}$, Straub RH, et al. Synovial fluid estrogens in rheumatoid arthritis. Autoimmunity Reviews. 2004 Mar 1; 3(3): 193-8. https://doi.org/10.1016/j.autrev.2003.08.003 PMid:15110231

13. Islander U, Jochems C, Lagerquist MK, Forsblad-d'Elia H, Carlsten H. Estrogens in rheumatoid arthritis; the immune system and bone. Molecular and Cellular Endocrinology. 2011 Mar 15; 335(1): 14-29. https://doi.org/10.1016/j. mce.2010.05.018 PMid:20685609

14. Mur E, Hartig F, Eibl G, Schirmer M. Randomized double blind trial of an extract from the pentacyclic alkaloid-chemotype of Uncaria tomentosa for the treatment of rheumatoid arthritis. The Journal of Rheumatology. 2002 Apr; 29(4): 678-81. PMid:11950006

15. Uncaria tomentosa (cat's claw) improves quality of life in patients with advanced solid tumors. - PubMed - NCBI [Internet]. [cited 2018 May 13]. Available from: https://www. ncbi.nlm.nih.gov/pubmed/25495394

16. Araujo S, Carmo M do, Farias IL, Gutierres J, Dalmora SL, Flores N, et al. Uncaria tomentosa - Adjuvant Treatment for Breast Cancer: Clinical Trial [Internet]. Evidence-Based Complementary and Alternative Medicine. 2012. [cited 2018 May 13]. Available from: https://www.hindawi.com/journals/ ecam/2012/676984/ 